\title{
Proceso de empoderamiento personal para mujeres en condiciones de violencia por parte de su pareja. Una experiencia de extensión universitaria entretejiendo la docencia
}

\section{Personal Empowerment Process for Women in Domestic Violence Conditions. A College Experience Intertwined with Teaching}

Celia Ma. Víquez Rojas

Universidad Nacional

Escuela de Psicología Heredia, Costa Rica celia.viquez.rojas@una.cr

Karla Cuyuch Artiga

Universidad Nacional

Heredia, Costa Rica info@karlademontagne.com

Natalia María Argüello Borbón

Universidad Nacional

Heredia, Costa Rica naty12_cr@hotmail.com

Recibido: 7/05/2020 Aceptado: 17/08/2020

Resumen. Durante el segundo ciclo del 2017, dentro del Programa de Promoción y Atención a la Salud: Aportes desde la Psicología, de la Escuela de Psicología de la Universidad Nacional de Costa Rica, se implementó una experiencia dirigida a contribuir con el empoderamiento personal de mujeres del cantón de Heredia, que viven o han vivido violencia por parte de su pareja. Esta experiencia permite reconocer cómo una práctica de extensión universitaria se vincula directamente con la docencia. La articulación de estos componentes facilita el fortalecimiento de las capacidades del estudiantado y atiende una necesidad concreta de la comunidad herediana en materia 
Revista Universidad en Diálogo • Vol. 10, N. ํ 2, Julio-Diciembre, 2020 • 79-95

ISSN 2215-2849 • EISSN: 2215-4752

URL: https://www.revistas.una.ac.cr/index.php/dialogo/index Correo eleCtrónico: universidadendialogo@una.ac.cr

DOI: https://doi.org/10.15359/udre.10-2.4

de salud. La violencia de género contra las mujeres por parte de sus parejas actualmente constituye un problema de salud pública que ha venido en aumento, y ante el que la academia y la psicología tienen un rol incuestionable para su estudio y disminución. A partir de este reconocimiento es que surge la iniciativa que se concreta con la implementación de un proceso guiado por una propuesta metodológica participativa, tipo taller, con enfoque género-sensitivo; construida y facilitada conjuntamente por dos estudiantes del curso Modelos de Intervención en Procesos Grupales, del último nivel de licenciatura de la carrera de Psicología, y la académica responsable de la actividad. El proceso de empoderamiento estuvo encaminado a la promoción, al desarrollo y al fortalecimiento de capacidades y habilidades psicosociales que les permitan a las mujeres participantes trabajar por una vida saludable, libre de violencia. Esta experiencia facilitó cambios significativos en las creencias acerca del rol tradicional femenino, de su lugar en la relación de pareja; contribuyó con la resignificación de sus experiencias de violencia, valorando sus aciertos y reconociendo sus retos, y también favoreció la toma de decisiones y la responsabilidad sobre su estabilidad emocional.

Palabras clave: empoderamiento, género, violencia contra las mujeres.

Abstract. During the 2017 second term, the School of Psychology of the National University of Costa Rica implemented an experience aimed at contributing to the empowerment of women in the Heredia Province, specifically those who had lived or are living in situations of violence by their intimate partners. The experience was part of the Program for Health Promotion and Awareness: contributions from Psychology. This experience intended to show how academic activity directly relates to teaching practices. The articulation of these components helped strengthen the students' capabilities and tried to meet a concrete necessity of the Heredia community in health-related matters. Gender violence against women, particularly from their intimate partners, currently represents a growing public health issue; psychology and academia have an unquestionable role to play to study and reduce it. From this, the initiative emerged when implementing a process guided by a participative-methodology proposal. The experience was designed as a workshop focused on gender and built and enabled by the corresponding teacher and students of the course "Modelos de Intervención en Procesos Grupales" (Intervention Models in Group Processes), normally taught in the last year of the bachelor's degree in psychology. The empowerment process aimed to promote, develop, and strengthen psychosocial aptitudes and skills that allow the participating women to work for a healthy, free-of-violence life. This experience enabled significant changes in women's beliefs about traditional gender roles, and their place in their sentimental relationship. It helped them redefine their experiences with violence and allowed them to value their accomplishments and reassess their mistakes; it also favored their decision-making and their responsibility for their emotional stability.

Keywords: empowerment, gender, violence against women. 
URL: https://www.revistas.una.ac.cr/index.php/dialogo/index

CORREO ELECTRÓNICO: universidadendialogo@una.ac.cr

DOI: https://doi.org/10.15359/udre.10-2.4

\section{Apreciaciones introductorias}

El Programa de Promoción y Atención a la Salud: Aportes desde la Psicología, de la Escuela de Psicología de la Universidad Nacional de Costa Rica, vigente a partir del año 2016, nace como parte del proceso de reflexión y madurez de la Unidad Académica, que reconoce la necesidad de nuevos rumbos relativos a la trascendencia de su contribución a la comunidad universitaria y a la sociedad costarricense.

Uno de los pilares del Programa consiste en promover una formación integral del estudiantado de psicología, estableciendo espacios de crecimiento personal que se acompañen de una oferta académica pertinente, reconociéndoles como agentes de la salud, desde la promoción y la atención. Además, busca, entre otros objetivos, desarrollar estrategias de intervención a lo interno y a lo externo de la universidad que favorezcan la salud de las personas y contribuir con su bienestar.

Es por medio de la articulación de los componentes de extensión y docencia que se concretan iniciativas con las que se pueden fortalecer las capacidades del estudiantado y atender necesidades de la comunidad nacional en materia de salud. Se realizan abordajes individuales y grupales, asumiendo el compromiso de formar profesionales capaces de favorecer la transformación de las sociedades en las que participan. Para lograr esto se requiere un verdadero sentido de responsabilidad en relación con la construcción del conocimiento, así como con la puesta en marcha de acciones concretas que impliquen contribuciones significativas, desde la psicología, a la salud integral de las personas y las comunidades. ${ }^{1}$

En este marco es que surge la iniciativa de desarrollar un proceso de empoderamiento dirigido a mujeres de la comunidad herediana, sobrevivientes o en condiciones de violencia por parte de la pareja, que se realizó durante el II ciclo del año 2017, dirigido por dos estudiantes del último nivel de la carrera, quienes deciden desarrollar, en este espacio, la práctica profesional correspondiente al curso Modelos de Intervención en Procesos Grupales de la Licenciatura en Psicología, con el acompañamiento y la supervisión de la académica participante en el Programa, responsable de esta actividad.

Desde el Programa se valora y se reconoce la necesidad de brindar apoyo a la comunidad herediana, específicamente a mujeres en condiciones de violencia

\footnotetext{
Programa de Promoción y Atención a la Salud: Aportes desde la Psicología, de la Escuela de Psicología de la Universidad Nacional de Costa Rica
} 
por parte de sus parejas, con el fin de colaborar en el mejoramiento de la calidad de su vida, reconociendo que como Unidad Académica se cuenta con recursos profesionales especializados, con estudiantado capacitado y con una infraestructura adecuada.

Esta decisión reposa en la realidad que evidencian las cifras de la violencia contra las mujeres que se han venido reportando en Costa Rica, y especialmente en la responsabilidad social que, como academia y disciplina psicológica, nos corresponde ante una problemática de salud pública y de violación de los derechos de las mujeres (Organización Mundial de la Salud, 2017), que ha venido en aumento; el rol que tenemos en su estudio y disminución es incuestionable.

De acuerdo con el Observatorio de Violencia de Género Contra las Mujeres y Acceso a la Justicia, en Costa Rica ocurren 2 femicidios por mes y 132 solicitudes de medidas de protección se presentan por día, solicitudes realizadas por mujeres. Y desde el $2007^{2}$ al 2017 se contabilizó un total de 313 femicidios. Para el año 2018, se reportaron 24, con oleadas de dimensiones significativas.

El femicidio constituye la manifestación más grave de las violencias de género contra las mujeres, en la que la mujer, por el hecho de serlo, es asesinada a manos de su pareja actual o pasada, o bien de un hombre con quien no tiene o tuvo ninguna forma de relación. El femicidio refleja el acto más lamentable de las relaciones desiguales entre mujeres y hombres.

La violencia contra las mujeres afecta de forma negativa su salud mental, emocional, física y sexual, y de acuerdo con la OMS (2017) existen "datos que demuestran que las intervenciones que promueven la sensibilización y emancipación de la mujer, la prestación de orientación psicológica (...) podrían favorecer la prevención o la reducción de la violencia de pareja contra la mujer".

Entonces, con base en este panorama, se promueve la creación de un espacio dirigido al empoderamiento de mujeres de la comunidad de Heredia, que se encuentren en condiciones de violencia por parte de la pareja, o bien, vivan las secuelas de la misma; con el objetivo de favorecer el desarrollo y fortalecimiento de capacidades y habilidades psicosociales que les permitan trabajar por una vida saludable, libre de violencia.

Para efectos de este proceso, tomamos como referente el concepto que comparte Maton (2008) sobre empoderamiento, planteado como "un proceso 
URL: https://www.revistas.una.ac.cr/index.php/dialogo/index

CORREO ELECTRÓNICO: universidadendialogo@una.ac.cr

DOI: https://doi.org/10.15359/udre.10-2.4

en grupo, participativo y de desarrollo, a través del cual individuos y grupos marginalizados $\mathrm{u}$ oprimidos obtienen un mayor control sobre sus vidas $\mathrm{y}$ sobre el medio, adquieren recursos valiosos y derechos básicos, alcanzan metas importantes en sus vidas" (p. 5). Rescatando la importancia de que las mujeres participantes logren conseguir un nivel de autonomía tal que les permita apropiarse de decisiones personales saludables a favor de una vida libre de violencia.

\section{¿Cómo desarrollamos la iniciativa?}

Como primer momento, realizamos una revisión documental sobre la violencia contra las mujeres por parte de sus parejas, y de experiencias grupales para el empoderamiento femenino con enfoque de género, reconociendo en cada paso de la estrategia metodológica la extensa práctica profesional en materia de violencia contra las mujeres de la académica responsable de la actividad. En este punto, el material bibliográfico consultado nos permite definir y sustentar los conceptos que facilitan el marco referencial desde el cual realizamos nuestra intervención.

Iniciamos diseñando una propuesta de intervención grupal que permitiera el cumplimiento del objetivo de trabajo con las mujeres. Planteamos una metodología participativa, tipo taller, con enfoque género sensitivo; ${ }^{3}$ elegimos técnicas que consintieran la construcción conjunta del conocimiento y un proceso abierto hacia el empoderamiento personal, promoviendo el análisis, la reflexión y la toma de sus propias decisiones (Abarca, 2016).

Con esta metodología buscamos generar una dinámica en la que las mujeres participantes le dieran contenido al proceso y también respuesta a sus inquietudes, rescatar el valor de sus pensares, sentires y experiencias, y reconociendo su cuerpo como territorio de su pertenencia; además, que las mujeres identificaran y practicaran, grupal e individualmente, capacidades y habilidades que les facilitaran una vida saludable, sin violencia. De igual forma, correspondía crear las condiciones necesarias para el apoyo y la contención emocional como grupo y desde nosotras, como facilitadoras del proceso.

\footnotetext{
Esta perspectiva permite explicarnos y comprender la construcción simbólica de las mujeres y los hombres en un entramado de relaciones de poder desigual de los unos sobre las otras. Nos permite reconocer que esta diferenciación sexual ha marcado históricamente la discriminación y la violencia contra las mujeres, atravesadas por una cultura patriarcal. Elaboración propia basada en la revisión de material bibliográfico, considerando las siguientes autoras: Marcela Lagarde, 1996, y Ma. Cristina González, 2014.
} 
Partimos de la premisa de que un proceso de empoderamiento personal puede generar cambio si se construye desde tres niveles: lo cognitivo, lo emocional y la experiencia, por lo que cada una de las dinámicas o técnicas diseñadas tuvieron un espacio para la reflexión y el análisis, un momento para sentir y otro para construir alternativas de prácticas cotidianas que promuevan una vida sin violencia.

Otra de las consideraciones que tomamos en cuenta en el diseño y puesta en ejecución de la propuesta metodológica es la flexibilidad en el manejo de la misma, es decir, contemplamos la posibilidad de realizar ajustes requeridos a partir de las necesidades específicas de las participantes, tales como el nivel socioeconómico, educativo, discapacidad y la severidad del maltrato.

De igual forma, contemplamos los factores protectores necesarios dirigidos a prevenir, en tanto sea posible, que las mujeres sufran nuevos incidentes de violencia, además, tomamos las previsiones requeridas para evitar que se diera algún tipo de sometimiento de la mujer al trauma, o bien, que se realizaran revictimizaciones a partir de relatos repetidos e innecesarios sobre las situaciones de abuso. Asimismo, se reconoció como aspecto clave evitar que las mujeres participantes revivieran o se refirieran a situaciones que no podían ser abordadas durante el proceso grupal.

El proceso lo estructuramos para diez sesiones, desarrolladas semanalmente, de dos horas y treinta minutos cada una, lo que supone un total de treinta y dos horas de trabajo grupal con las mujeres participantes. Todo el proceso se realiza en el Edificio de Usos Múltiples de la Universidad Nacional, instalaciones en las que trabaja la Escuela de Psicología.

\section{Contenidos temáticos y estrategias metodológicas}

Para cada una de las sesiones trabajamos aspectos conducentes a colaborar en el proceso de empoderamiento de las mujeres participantes, así que:

- En la primera sesión, recibimos y damos la bienvenida a las participantes, les compartimos la forma en la que se desarrolla el proceso, rescatando su lugar como el centro del mismo, la apertura y la flexibilidad que acompañan cada una de las sesiones. Realizamos una dinámica de presentación para promover un acercamiento humano entre quienes participamos, así como un adecuado clima de 
confianza en el grupo. En esta sesión iniciamos con la aproximación al derecho humano a una vida sin violencia, a la construcción de las identidades de género y los efectos en sus cotidianidades como mujeres. También se disponen espacios para fomentar la esperanza y la posibilidad de cambio.

- Durante la segunda sesión abordamos, desde una perspectiva crítica, las feminidades tradicionales, empleando técnicas concretas que vinculan con la vida diaria, por ejemplo, la escucha, el análisis y la reflexión sobre canciones que típicamente oyen y que ellas mismas aportan. Otra de las técnicas que se emplean para este abordaje es que cada participante identifica aquellos mandatos sociales que más le pesan y los asocia con un lugar de su cuerpo que le sea significativo en función de ese mandato. También, exploramos con las participantes el concepto de patriarcado y su lugar en el proceso de construcción de las identidades de género y el manejo del poder en las relaciones intergenéricas e intragenéricas, para lo que empleamos técnicas participativas y recursos de apoyo audiovisual que promuevan la reflexión en relación con sus propias vivencias.

- Las tres sesiones siguientes nos centramos en reflexionar y comprender, a partir de sus experiencias personales compartidas de forma voluntaria, la dinámica de la violencia contra las mujeres por parte de sus parejas, sus manifestaciones y las implicaciones en su salud emocional, mental y física. Las técnicas empleadas van dirigidas a promover la escucha propia y mutua, la contención emocional como grupo, la validación de sentimientos y la desculpabilización, potenciando la capacidad resiliente en lo individual y en lo colectivo.

- En la sesión seis abordamos el ciclo de la violencia y el lugar de la dependencia que se genera en el mismo, reconociendo el efecto en sus propias vidas, pero respetando y valorando las capacidades de las mujeres participantes a pesar de las secuelas de la violencia. En esta sesión también abrimos espacios para compartir, de forma voluntaria, experiencias personales de violencia por parte de su pareja, la escucha y la contención emocional reposa en el grupo. En esta sesión, como en las anteriores, es clave el rescate de los pensares, los sentires y los haceres de las participantes. 
- Para las dos sesiones siguientes trabajamos en el reconocimiento y la validación de las estrategias empleadas para enfrentar la violencia, así como en la construcción de estrategias alternativas dirigidas a la promoción de una vida más saludable. Desarrollamos técnicas que fomentan la resignificación de sus propios cuerpos, la importancia de la puesta de límites en el proceso de empoderamiento, el reconocimiento y la validación de sus emociones, así como la oportunidad de responsabilizarse emocionalmente de sí mismas.

- Durante las dos últimas sesiones nos ocupamos de identificar con las mujeres participantes sus redes y recursos de apoyo, también trabajamos en la construcción de planes de seguridad personal que las alerten de involucrarse, o bien, retirarse de relaciones en las que puedan vivir violencia. Como parte del cierre del proceso realizamos técnicas que buscan la identificación de las fortalezas y los retos personales, así como también a nivel grupal, reconociendo el valor de las otras como nuevas redes de apoyo. También realizamos técnicas dirigidas al cierre emocional del proceso, que facilitan reflexionar y compartir logros y experiencias significativas a partir de los aprendizajes, las vivencias compartidas y el apoyo mutuo.

\section{El camino recorrido}

Una vez diseñada la propuesta metodológica, nos abocamos a la tarea de definir los pasos a seguir en el trabajo de campo. El primer aspecto es la definición de día, horario y lugar específico, una vez contemplado esto, planteamos la convocatoria, a cargo de las dos estudiantes que participan de forma directa en el desarrollo del proceso, ellas se dan a la tarea de idear un nombre, ${ }^{4}$ de diseñar un afiche, ubicarlo en lugares estratégicos del cantón central de Heredia y compartirlo por redes sociales.

También planteamos una ficha para la entrevista telefónica previa a la inscripción, con la que buscamos identificar características clave del grupo con el que trabajamos y reconocer si alguna mujer se encuentra en una situación de violencia de alto riesgo, ante lo que es necesario tomar previsiones al respecto (ver figura 1). Además de considerar los criterios de inclusión para participar en el proceso: ser mujer, haber vivido o vivir actualmente situaciones de violencia por parte de la pareja y ser mayor de edad. Definimos como cupo máximo quince personas, ya que es un número que permite un manejo grupal adecuado sin descuidar los espacios individuales.

Taller de empoderamiento Porque Merezco una Vida Sin Violencia. 
URL: https://www.revistas.una.ac.cr/index.php/dialogo/index

CORREO ELECTRÓNICO: universidadendialogo@una.ac.cr

DOI: https://doi.org/10.15359/udre.10-2.4

Figura 1. Ficha de entrevista telefónica

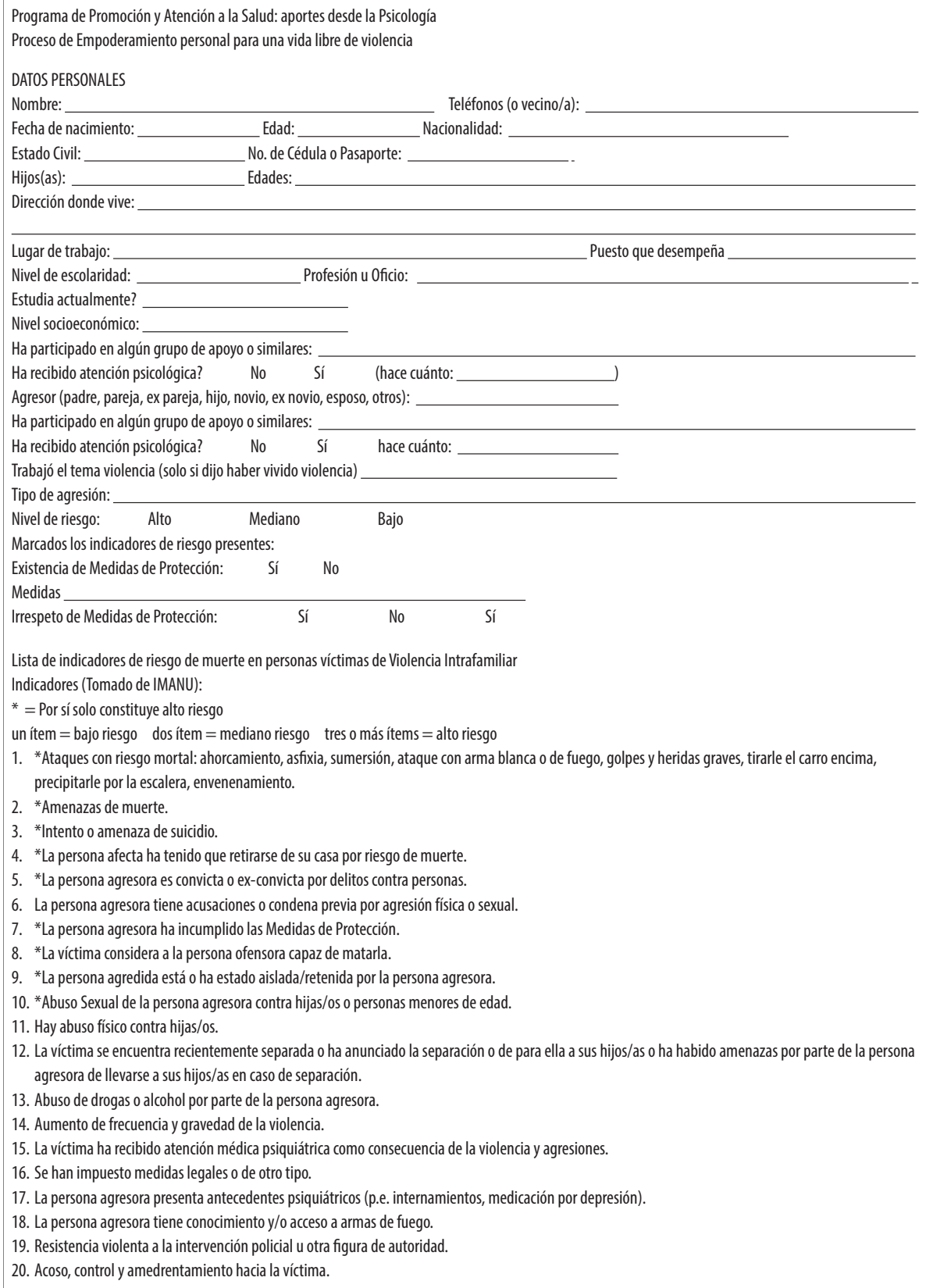


Mientras se da el período de inscripción, preparamos los materiales requeridos para cada sesión, una vez concluido el proceso de inscripción, identificamos que, si bien todas las mujeres tienen condiciones de violencia, ninguna se ubica en alto riesgo.

Iniciamos el proceso de empoderamiento que dura diez sesiones, durante el cual debemos tomar una postura flexible, acogedora y de contención para con el grupo y las mujeres en lo individual. También abrimos un grupo en WhatsApp, previa autorización de las participantes, con el fin de comunicarnos de forma más fluida, pero sobre todo como un espacio que pueda constituir el mecanismo tecnológico que sostenga comunicado al grupo, una vez concluido el proceso.

Seis meses después de concluido el proceso de empoderamiento, invitamos a las participantes a un encuentro, como un espacio de seguimiento y reconocimiento de los efectos que puede haber tenido en las mujeres ser parte de la experiencia. Utilizamos un grupo focal para compartir vivencias, pensares y sentires, así como los cambios conseguidos, identificados por ellas mismas en cuanto a procurar para sí una vida saludable, sin violencia.

\section{¿Con quiénes trabajamos?}

Se inscribieron un total de quince mujeres, con edades desde los veintidós hasta los sesenta y seis años, con niveles educativos que van desde primaria incompleta hasta universitaria incompleta, la mayoría casadas o en unión libre, solo una no tiene hijos ni hijas. Diez son vecinas de la comunidad herediana, dos de Alajuela y tres de San José, a quienes les abrimos el espacio, dado su nivel de interés y por considerarlo un derecho. De todas ellas, catorce expresan haber vivido experiencias de violencia por parte de su pareja (esposo o novio), y algunas se encuentran en esa relación.

Siete de las participantes inscritas no asisten al primer encuentro, las contactamos, sin embargo, no asisten a ninguna sesión. La cantidad de mujeres que participan del proceso son un total de ocho, pero una de ellas debe retirarse por motivos laborales.

\section{¿Y sobre los resultados obtenidos a partir de la implementación del proceso?}

El proceso grupal evoluciona de forma favorable durante todas las sesiones, con un nivel satisfactorio de asistencia y participación activa en cada una de 
URL: https://www.revistas.una.ac.cr/index.php/dialogo/index

CORREO ELECTRÓNICO: universidadendialogo@una.ac.cr

DOI: https://doi.org/10.15359/udre.10-2.4

las sesiones, lo que facilita que la dinámica procesal del grupo sea más fluida. La secuencia de los contenidos planteados para el desarrollo del proceso facilita un encadenamiento paulatino y positivo en la reflexión, el análisis y la construcción de conocimientos, así como en el acercamiento respetuoso a las vivencias dolorosas de las participantes.

Los resultados los planteamos reconociendo lo acontecido durante el proceso de empoderamiento, y lo rescatado por las participantes en el espacio de encuentro realizado seis meses después.

\section{Reconstruyendo mi identidad femenina}

La identidad constituye ese conjunto de pensamientos, representaciones y afectos que una persona tiene sobre sí misma y sobre las demás personas (Lagarde, 1994), y es la sociedad, desde lo privado y lo público, la que ha construido sistemas de creencias desde los que se definen los deber ser, sentir y hacer para las mujeres y para los hombres.

La concepción sobre el ser mujer que las mujeres plantean tener antes de vivir el proceso de empoderamiento hace referencia a visualizarse a sí mismas como objetos sexuales, destinadas al servicio de los otros, a los oficios domésticos, al cuido, a la maternidad y a la complacencia de su familia, lo que vemos reflejado en frases expresadas por las participantes:

"En mi caso, un objeto sexual, soy a la que le toca lavar, cocinar... ser, qué se yo, ama de casa". "Soy la que lava, soy la que plancha, soy la que cocina, o sea, era la soy de todo". "Soy ese ser servil, la madre, la esposa, la hija, perfecta". "Soy la niñera, la que cuida a quien lo necesite, mis necesidades van luego". (Extractos de testimonios de participantes, 2017)

Reconocemos aquí características propias del rol tradicional femenino, el ser para otros, sustento de otros, quienes constituyen el centro de sus vidas, de sus pensamientos y de sus afectos, es decir, la subjetividad de las mujeres ha sido construida para ser en función de las demás personas, debe cuidar, proteger y nutrir a otros. Todo lo cual es aprendido y fortalecido en su proceso de socialización, para ser reconocida como mujer debe pensar en otros primero antes que en ella misma. Ver recuadro 1. 
Recuadro 1: Módulo básico para sentir, pensar y enfrentar la violencia de género, intrafamiliar y sexual

"Se fomenta la generosidad, la receptividad, la incondicionalidad hacia otros(as) y no se deja tiempo para el desarrollo de la autonomía y la independencia personal de las mujeres". (INAMU, 2006)

En el encuentro posterior pudimos escuchar algunos relatos que involucran creencias y vivencias que nos permiten reconocer cambios significativos para las participantes, en cuanto a su identidad como mujeres, redefiniendo su lugar en sus propias vidas.

"Ver mi vida para mí, esas personas son complemento en mi vida, pero no mi vida [refiriéndose a la familia]". "Estoy haciendo las cosas para mí, ahora yo me siento con libertad". "Yo les digo a mis hijos que no tengo que estar cambiando mantillas [a sus nietas]". "Ya todo es diferente, ya ahora no soy yo que tiene que ver cómo se componen las cosas". "En un principio me sentía un poquito incómoda porque estaba acostumbrada a hacer todo [para los demás], pero ahora me siento muy bien". (Extractos de testimonios de participantes, 2017)

\section{Resignificando mi transitar por la violencia}

La violencia contra las mujeres la entendemos como una clara violación de los derechos humanos, producto de una organización social sexista, en la que el abuso y el maltrato contra las mujeres es producto de relaciones de poder históricamente desiguales entre hombres y mujeres (Inamu, 2014). Específicamente, y de acuerdo con la Convención Belém do Pará (19945), "debe entenderse por violencia contra la mujer cualquier acción o conducta, basada en su género, que cause muerte, daño o sufrimiento físico, sexual o psicológico a la mujer, tanto en el ámbito público como en el privado" (art. 1).

La violencia por parte de la pareja se enmarca dentro de lo que se conceptualiza como la violencia doméstica, la que se refiere a todo aquel acto u omisión que tenga como resultado intencional un daño a la integridad física, sexual,

Adoptada por el Estado costarricense a partir de 1995, año en el que el país ratificó esta convención. 
URL: https://www.revistas.una.ac.cr/index.php/dialogo/index

CORREO ELECTRÓNICO: universidadendialogo@una.ac.cr

DOI: https://doi.org/10.15359/udre.10-2.4

emocional o social de un ser humano, y que entre las personas involucradas exista un vínculo familiar o íntimo (Claramunt, 2007).

En el grupo de mujeres participantes observamos historias de violencia por parte de sus parejas, relatadas con dolor y decepción, teñidas de miedo y soledad. Describen vivencias de violencia psicológica, física, sexual, durante su relación de pareja, reconociendo el impacto en su salud en general, plantean experimentar, entre otros síntomas, gastritis, colitis, ansiedad, trastornos en el sueño y la alimentación, problemas de autoaceptación, dificultad importante para poner límites y tomar decisiones, todo lo cual se acompaña de culpa y vergüenza:

"Bueno, él me pegó y yo llamé a la policía y se lo llevaron". "Me daba vergüenza que la gente supiera que yo era agredida y nunca lo admití". "Sentía temor por externar algo". "Por 20 años escondí que era víctima de violencia, sentía culpa por decir eso de mi marido". (Extractos de testimonios de participantes, 2017)

En el relato de las participantes también pudimos identificar el fenómeno intergeneracional de la violencia,${ }^{6}$ cuando las participantes hacen referencia a sus experiencias de violencia durante la infancia, también en expresiones de sorpresa cuando reconocen, hasta ese momento, eventos vividos como violencia, es decir, normalizaron la violencia. Ver recuadro 2.

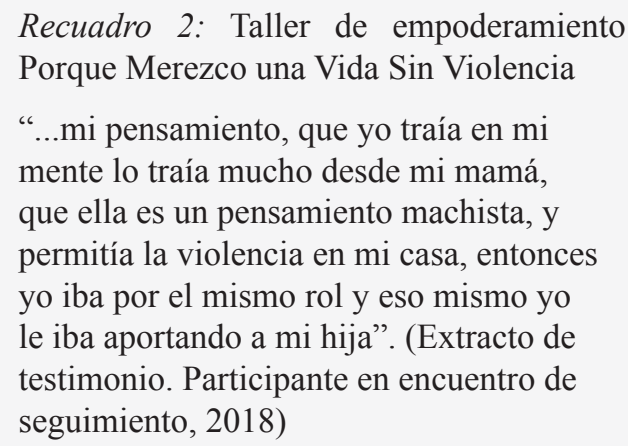

Podemos decir que la violencia constituye un fenómeno que trasciende generaciones, muchas mujeres y muchos hombres que han sufrido algún tipo de violencia durante su infancia pueden considerarla parte intrínseca de la vida, normalizándola al punto de no poder reconocerla, incluso, reproduciéndola. 
Posteriormente, logramos reconocer cambios en la forma en la que significan sus experiencias de violencia, rescatan las estrategias empleadas para sobrevivir a los malos tratos y a las agresiones por parte de su pareja. Identifican las capacidades que desarrollaron en esas condiciones de vida y las valoran como de su pertenencia y que las pueden emplear en su propio beneficio y bienestar.

\section{Una nueva mirada}

Poder verse, verse a sí mismas con una nueva mirada, como una mujer con valía propia, con capacidades y habilidades, con mayores niveles de autonomía y con el derecho de una vida sin violencia, fue uno de los resultados más significativos de este proceso de empoderamiento.

Reconocer su poder en la toma de decisiones y en su estabilidad emocional e identificar las estrategias empleadas para sobrevivir a la violencia como de su propia pertenencia y que pueden reorientarlas para conseguir colocarse a sí mismas en un lugar diferente en sus relaciones con los otros son algunos de los aspectos que identificamos en los discursos de las participantes cuando se refieren a los logros conseguidos a partir de vivir la experiencia del proceso.

"A partir de ese momento, de ese taller, vea usted que estoy separada de mi esposo, vivo muy bien, estoy muy contenta... y mire, no volví al hospital, la presión y todo se me reguló. Entonces estoy sí estoy muy bien, y digo yo: ¡qué maravilloso!". "No, no pienso volver con mi esposo, él es un agresor. No, ¿para qué? Yo no necesito volver a ese lugar, yo estoy muy bien como estoy". (Extractos de testimonios de participantes, 2018)

La mayoría de las participantes considera que se ve a sí misma con mayor libertad, con capacidad de decir lo que siente y lo que piensa y de poner límites, plantean que se sienten menos temerosas, que ya no sienten vergüenza por sus historias de violencia. Poder compartir con otras sus vivencias de agresión les permite verse a sí mismas y a las otras desde el afecto y la sororidad, ${ }^{7}$ generando lazos que facilitan la recuperación emocional y logran que la culpa se asome con menos frecuencia.

"Es una experiencia de las mujeres que conduce a la búsqueda de relaciones positivas y a la alianza existencial y política, cuerpo a cuerpo, subjetividad a subjetividad con otras mujeres, para contribuir con acciones específicas a la eliminación social de todas las formas de opresión y al apoyo mutuo para lograr el poderío genérico de todas y al empoderamiento vital de cada mujer" (Lagarde, 2006). 
URL: https://www.revistas.una.ac.cr/index.php/dialogo/index

CORREO ELECTRÓNICO: universidadendialogo@una.ac.cr

DOI: https://doi.org/10.15359/udre.10-2.4

En general, pudimos identificar que las participantes logran cuestionar su rol tradicional como mujeres, empezar el proceso de desaprender pensares, sentires y haceres que direccionan a experiencias de violencia, para aprender formas de mirarse más saludables y justas para consigo mismas. Reconocen que los cambios no son fáciles y que existe presión familiar para que se queden en el mismo lugar, pero mencionan haber comprendido que tienen el poder para decidir: "Entonces dicen que me volví peleona, pero lo que hago es recalcarles que yo tengo derecho a opinar, y a hacerlo y decirlo" (extracto de testimonio de participante, 2018).

Compartimos algunos de los relatos de las mujeres participantes en el encuentro de seguimiento, que evidencian cambios a nivel cognitivo, emocional y en su proceder cotidiano, cambios dirigidos a la capacidad de tomar decisiones estratégicas en su vida, es decir, a su empoderamiento (Casique, 2010):

“...logré tener el poder, eso era lo que yo esperaba, el poder de enfrentarme a varias cosas, aún tengo mucho que trabajar, pero sí he logrado muchas cosas". "Esto [el taller] que me enseñó [fue] a liberarme, a tomar decisiones, a sentirme feliz". "Yo misma tengo que tomar la decisión, soy una mujer adulta y sé lo que quiero". "Tengo más concientización con otras [mujeres], tengo más empatía". "Me dijeron que si quería ir a trabajar, yo tuve miedo, pero yo sentía que lo necesitaba y ahora trabajo fuera de mi casa". (Extractos de testimonios de participantes, 2017)

De acuerdo con los resultados, el proceso constituye un espacio que abre posibilidades a las mujeres para releerse desde su propia mirada, de resignificar sus historias de vida teñidas de violencia y reposicionar su lugar en su presente. Ver recuadro 3.

Recuadro 3: Taller de empoderamiento Porque Merezco una Vida Sin Violencia

"Entonces poco a poco, paulatinamente, fuimos dándonos cuenta que nos tocaba hacer muchos cambios por nosotras. Nos dimos cuenta que era necesario salir de tanto mito y prejuicio que cargamos, porque solo nos han hecho daño. Paulatinamente, sesión por sesión fuimos reflexionando, aprendiendo y creciendo. ¿Saben? Ahora nos vemos con más cariño". (Extracto de testimonio. Participante en encuentro de seguimiento, 2018) 


\section{Conclusiones}

La experiencia implementada con un grupo de mujeres sobrevivientes de violencia por parte de sus parejas, para trabajar en su empoderamiento personal por una vida libre de violencia, la consideramos como exitosa. $\mathrm{Ha}$ brindado un espacio de reflexión, análisis y vivencias que contribuyen con la salud integral de las mujeres.

Las mujeres que participan del taller de empoderamiento Porque Merezco una Vida Sin Violencia consiguen, tanto en el proceso como en los resultados del mismo, desafiar su propio sistema de creencias que reposa en un sistema patriarcal, consiguen un mayor nivel de autonomía en la toma de decisiones, así como recursos y capacidades humanas y sociales para trabajar en el control de sus propias vidas. El proceso promueve el empoderamiento personal de las mujeres sobrevivientes de violencia.

En relación con la experiencia de extensión académica entretejiendo la docencia, es posible reconocer la importancia de esta vinculación en la formación profesional, en tanto el estudiantado puede ser co-constructor del conocimiento en la práctica y tiene la posibilidad de ser partícipe directo en el desarrollo de acciones concretas dirigidas a suplir necesidades de la realidad nacional.

En este caso, como estudiantes que forman parte del proceso, robustecen su marco referencial en el tema de la violencia contra las mujeres, prueban y aplican sus conocimientos teórico-metodológicos en cuanto al trabajo con grupos y reconocen la importancia de la mirada género-sensitiva en los procesos de empoderamiento. Además, realizan una revisión personal, crítica y reflexiva, privilegiando el desarrollo y fortalecimiento de valores y actitudes necesarias para lograr un desempeño profesional, ético y humano.

Es necesario que, desde la academia, específicamente desde la Escuela de Psicología de la Universidad Nacional, se asuma una posición de mayor incidencia en lo relativo a la violencia contra las mujeres, una problemática de salud pública. Nos corresponde, como disciplina de las ciencias sociales y de la salud, reconocer nuestro rol incuestionable en su estudio, así como en el desarrollo de acciones concretas, en extensión y en docencia, para minimizar el impacto en las subjetividades y contribuir con los esfuerzos para su erradicación. 


\section{Referencias bibliográficas}

Abarca, F. (enero-junio, 2016). La metodología participativa para la intervención social: reflexiones desde la práctica. Revista Ensayos Pedagógicos, XI(1).http://www.revistas.una.ac.cr/ensayospedagogicos

Casique, I. (2010). Factores de empoderamiento y protección de las mujeres contra la violencia. $\mathrm{http}: / / \mathrm{www}$. scielo.org.mx/pdf/rms/v72n1/v72n1a2.pdf

Claramunt, M. (2007). Casitas quebradas. San José: Euned.

Convención de Belem do Para. (9 de junio, 1994). Ciudad de Belem do Para, Brasil.

Escuela de Psicología UNA. (21 de mayo, 2015). Programa de Promoción y Atención a la Salud desde el Aporte de la Psicología. http://www.fcs. una.ac.cr/psicologia/doc_psicologia/Programa\%20aprobado.pdf

Inamu. (2014). Convención Belém do Pará. http://www.inamu.go.cr/belemdopara

Lagarde, M. (1994). Género e identidades. Metodología de trabajo con mujeres. Fundación para el Desarrollo Educativo y Tecnológico Comunitario. Ecuador: Unicef.

Maton, K. I. (2008). Empowering community settings: Agents of individual development, community betterment, and positive social change. American Journal of Community Psychology. https://www.academia. edu/31021861/Empowering_Community_Settings_Agents_of Individual_Development_Community_Betterment_and_Positive_ Social_Change

Organización Mundial de la Salud. (2017). Violencia contra las mujeres. Datos y cifras. https://www.who.int/es/news-room/fact-sheets/detail/ violence-against-women

Poder Judicial. (2018). Femicidio. Observatorio de Violencia de Género Contra las Mujeres y Acceso a la Justicia. https://observatoriodegenero. poder-judicial.go.cr/soy-especialista-y-busco/estadisticas/femicidio/ 